Acta Poetica 27 (2)

OTOÑO

2006

\title{
Testimonio y memoria en el relato histórico
}

\author{
Pilar Calveiro
}

En este texto se aborda el papel del testimonio y la memoria en la construcción del relato histórico de las atrocidades estatales ocurridas en el siglo xx. Se discute qué lugar deben ocupar: 1) la experiencia personal transmitida por los testigos y 2) la social procesada en los ejercicios de memoria, para la construcción de la historia "verdadera"; en otros términos, cuál es la "verdad" del testimonio y la memoria y, en consecuencia, hasta qué punto éstos deben permear la construcción histórica. El análisis se realiza desde el contexto de la experiencia argentina con respecto al terrorismo de Estado de los años setenta, como caso de los Estados latinoamericanos "desaparecedores" que intentaron borrar toda disidencia, pero también toda huella de su accionar represivo. En este sentido, el testimonio y la memoria se organizaron desde el inicio como prácticas signadas políticamente, múltiples pero con una direccionalidad específica: la resistencia al silencio oficial. Es cierto que el relato histórico, por su parte, no carece de posicionamiento. Sin embargo, debe dar cuenta de los diferentes sentidos que se manifiestan en una sociedad, no sólo de los resistentes. Se podría decir que la historia arma un relato más abarcador, de mayor generalidad, pero al hacerlo suele caer en la tentación disciplinar de pretenderse superior y de reivindicar un supuesto derecho de calificación y jerarquización de los saberes más apegados a la experiencia directa. Sin embargo, también tiene la posibilidad de construirse en articulación y diálogo con ellos, recuperándolos y confrontándose con ellos. La diferencia entre una y otra postura tiene una "valencia" política: el lugar que se le asignará a la resistencia en la construcción de la historia del terrorismo de Estado, como relato de "verdad" socialmente validado.

This text addresses the role of witnessing and memory in the construction of the historical account of state-inflicted atrocities in the 20th century. It discusses: 1) personal experience transmitted by witnesses and 2) social experience processed in memory for the construction of the "true" history. In other words, what is "true" in the testimonials and in memory and, consequently, to what extent they should influence the construction of history. The analysis is performed from the 
point of view of the Argentinean experience of State Terror in the '70s, as one of the cases of Latin-American states with "disappearances", which tried to eliminate every opposing opinion as well as all traces of the repression. In that sense, testimonials and memory were organized since the beginning as politically denoted practices, varied though carrying one specific direction: resistance to official silence. Although it's true that the historical account is not totally lacking in positioning, it should account for the different meanings evidenced in a society, not only in the members of the resistance. We could say that history builds a more encompassing and more general account though, in doing so, it usually falls for the disciplinary temptation of considering itself superior and of vindicating an alleged right of qualification and hierarchy over knowledge more connected to direct experience. However, it also has the possibility of being constructed in articulation and dialogue with this knowledge, by taking it into account and confronting it. The difference between these two possible approaches has a political "mark": the role assigned to resistance in the construction of the history of State Terror as a socially validated "true" account. 
Pilar Calveiro

Benemérita Universidad Autónoma de Puebla

\section{Testimonio y memoria en el relato histórico}

Los genocidios, las masacres, los crímenes de lesa humanidad perpetrados por los Estados son conocidos por sus contemporáneos pero negados cínicamente por los responsables y por buena parte de la sociedad que los "desconoce". El siglo Xx dio muchísimos ejemplos de ello, en el mundo y en América Latina.

Los hechos atroces, que el Estado y sus sociedades niegan —no por desconocimiento sino más bien para eludir sus respectivas responsabilidades-, se imponen como presencia incómoda a través del relato de los sobrevivientes de las masacres que, sin embargo, tarda en encontrar oídos dispuestos a escuchar. Sin embargo, poco a poco y sólo si las relaciones sociales de poder se modifican, los testimonios se abren paso a través del silencio, lo van rompiendo y colocan los hechos atroces, de manera ineludible, bajo la mirada de sus contemporáneos.

Es a partir de estos primeros relatos de lo atroz que esas mismas sociedades desandan el camino del silenciamiento y el olvido para emprender el difícil trabajo de la memoria que, en verdad, siempre ha estado ahí aunque de otra manera. Y es que la memoria que comienza a tejerse desde el impacto de lo 
testimonial es otra, es la que recuerda —en contra del discurso predominante del orden y la subversión - el alma violenta, ilegal e ilegítima de esos Estados, la impunidad del poder, las aventuras y desventuras de las resistencias y, sobre todo, su horizonte pasado y especialmente futuro de posibilidad.

Así, frente al texto discursivo del Estado comienza a tejerse otro que entra en contradicción con él, que lo desmiente y que disputa las características que posteriormente deberá tener el relato histórico, con las pretensiones de "verdad" propias del discurso científico. Se trata de una verdad que se reconoce construida, relativa, pero que finalmente siempre esgrime su "superioridad" disciplinar y sistemática.

Estos tres momentos: el testimonio como ruptura del silencio, la memoria como trama de los relatos de la resistencia y la historia como texto estructurador de alguna verdad, sea o no oficial, han estado presentes en el proceso de revisión de las atrocidades estatales que se han logrado exhibir y denunciar - porque hay otras que permanecen ocultas e impunes por muchísimo tiempo- - En dicho proceso, una de las discusiones decisivas es qué lugar deben ocupar 1) la experiencia personal transmitida por los testigos y 2) la social procesada en los ejercicios de memoria, dentro del relato histórico "verdadero"; en otros términos cuál es la "verdad" del testimonio y la memoria y, en consecuencia, hasta qué punto deben permear la construcción histórica.

Así ha sucedido con los genocidios judío y gitano, perpetrado por los nazis, y también así está sucediendo en toda América Latina, en relación con el exterminio de la disidencia política practicada por los Estados en los años setenta, mediante la práctica institucional de la "desaparición" forzada de personas.

El caso argentino es, de alguna manera, el trasfondo de la discusión que aquí se presenta. Sin embargo, considero que éste, por inscribirse en una política continental de características hasta cierto punto semejantes, tiene una proyección que 
alcanza a otras realidades latinoamericanas. En consecuencia, pasaré a puntualizar algunas de sus características principales.

En Argentina, la salida del gobierno militar ocurrió en una situación de debilitamiento de la cúpula militar dentro de las estructuras de poder, que no estoy en condiciones de analizar en este texto. Sin embargo, parece claro que las Fuerzas Armadas, como grupo dirigente, habían extraviado toda legitimidad en virtud de varios factores, entre los que se puede señalar: 1) una represión brutal, a todas luces ilegal, que hizo "desaparecer", sin dejar rastro visible, a cerca de 30000 personas desatando campañas de cuestionamiento nacionales e internacionales, 2) un proyecto económico fuertemente impopular, que enfrentaba una resistencia creciente, y 3 ) una guerra perdida - la de Malvinas-, que puso de frente a los militares no sólo con su incapacidad política y económica sino con la ineptitud demostrada en el área más específica de su competencia: la militar. Esta situación de pérdida de reconocimiento del papel dirigente que habían ostentado en la estructura de poder permitió que, casi inmediatamente de su salida del gobierno (1983), se iniciara el proceso de investigación que terminó, apenas dos años después en el Juicio a las Juntas (1985).

La preparación y la realización del juicio supusieron una avalancha de lo testimonial que puso ante los ojos de la sociedad las características que había tenido el modelo represivo de desaparición de personas, con todas sus atrocidades. La celebración misma del juicio y la condena a los comandantes colocó definitivamente todo eso, sabido-negado por la sociedad, en un lugar incuestionable: el de la verdad jurídica.

Una vez establecida la verdad de los hechos, de acuerdo con los recursos de prueba del derecho - coincidencia de los testimonios, entre otros - la sociedad pasó a la construcción de una memoria que implicó distintos debates: inocencia/participación 
y/o complicidad de la sociedad, responsabilidades compartidas y responsabilidades diferenciadas en los hechos de los setenta, identidad política y militancia de las víctimas, legitimidad e ilegitimidad de las prácticas armadas, entre otros.

Como es natural, la riqueza de este trabajo consistió en la multiplicidad de puntos de vista que, en ciertas ocasiones, el debate permitió acercar aunque en otras no. Sin embargo, siempre ayudó a profundizar la reflexión sobre lo vivido manteniendo la discusión y abriendo nuevas puntas de análisis, siempre muy apegadas a la experiencia testimonial de las víctimas, al relato de los diversos protagonistas y a las apreciaciones interpretativas de unas y otras, en un sentido que se fue ampliando progresivamente. Ciertamente, la sociedad toda era, de alguna manera, sobreviviente y protagonista y, en ese sentido, la memoria se fue construyendo como un trabajo a partir de la diversidad de experiencias vividas y sus diferentes interpretaciones. Sobrevivientes directos, madres y abuelas, hijos, pero también amigos, viejos compañeros de militancia, hijos de antiguos militantes, gente que había vivido los setenta y recordaba el miedo o los alegatos al silencio, jóvenes que nacieron después de los setenta pero que venían de familias que recordaban o bien de familias que no recordaban, y querían comprender ese silencio, en fin, no todos, pero muchos, emprendieron este trabajo de tomar su experiencia y tratar de articularla con otras, explicarla, comprenderla. Se multiplicaron así los organismos de defensa de los derechos humanos y las organizaciones dedicadas a distintas prácticas de la memoria con sus respectivos relatos. Creo que son pocas las sociedades que han desarrollado tan rápida y profusamente el trabajo de memoria.

De manera más reciente, ha iniciado otro debate: cuál es la "verdad" del testimonio, cuáles sus posibles sesgos y abusos y cuál es el uso que la historia, como relato interpretativo con pretensiones de "verdad", podría o debería hacer del material 
testimonial. A lo largo de las páginas que siguen trataré de argumentar, en un lenguaje estrictamente académico, la idea de que la articulación que el relato histórico logre con el material testimonial y los trabajos de la memoria es clave para la recuperación de la dimensión resistente y contrainstitucional de lo vivido por nuestras sociedades.

Los países latinoamericanos han abordado la memoria del terrorismo de Estado de los años setenta y ochenta con distintos niveles de reconocimiento del fenómeno, de los mecanismos de reparación en relación con las víctimas pero, sobre todo, con políticas diferenciadas pero por lo regular muy inconsistentes para la identificación y sanción de los responsables.

En este proceso, los testimonios de las víctimas son el primer paso que, junto a un trabajo más amplio de restitución de la memoria social, abren la posibilidad de procesos legales, las más de las veces muy limitados por el propio Estado. Treinta años después se ha recogido buena parte de los testimonios pero, en el contexto de las actuales democracias, los trabajos de la memoria no afectan de manera sustantiva a las actuales redes de poder que, en muchos casos, "lavan" sus caras permitiendo ciertas reparaciones — aunque casi todas ellas fijan límites muy precisos para los procesos legales contra los responsables - En este contexto, y a las puertas de la desaparición física de los ejecutores principales —lo que "resuelve" parte del problema porque permitiría una "condena" formal sin condena jurídica, una toma de distancia de procesos inaceptables sin asumir los costos políticos de su sanción- se impone ahora el debate de quién construirá el relato histórico de lo acontecido y cómo se hará. Es decir, quién y cómo se fijará la "verdad" histórica, acorde con las nuevas formas del poder, porque la historia que contaron los desaparecedores de los setenta - fueran civiles o militares, en gobiernos de facto o en "democracias" restringidas - ha caído inevitablemente en desuso 
y requiere de una actualización potable para las actuales formas de configuración del Estado.

La reorganización del poder político en las actuales democracias, fuertemente excluyentes, ${ }^{1}$ se articula con nuevas figuras del derecho, como la imprescriptibilidad de los delitos de lesa humanidad (pero por otro lado también la cancelación de derechos laborales) y reclaman la construcción de "discursos de verdad" propios, entre los cuales el relato histórico ocupa un lugar primordial para su validación. Una vez más, el triángulo poder-derecho-verdad, del que habló Michel Foucault, se constituye desde cada uno de sus vértices, que se reclaman recíprocamente. Creo que en este contexto, de reformulación de los discursos de verdad de un poder mutante, se deben inscribir las distintas reflexiones sobre la validez, los usos y los desusos del testimonio, la memoria y la historia.

Un elemento que, a mi juicio, enturbia esta discusión es que ésta se suele abordar sin localizaciones históricas y políticas específicas; se habla de "el" testimonio, "la" memoria y "la" historia, de manera que las apreciaciones se "alargan" tanto que, pretendiendo ser válidas en general, terminan resultando inútiles para la comprensión de los casos específicos. Para evitar este problema, en este texto me referiré al papel del testimonio, la memoria y la historia del terrorismo de Estado de los años setenta, es decir, al caso de Estados desaparecedores que intentaron borrar toda huella de su accionar represivo contra enemigos políticos como un acto de poder, frente al cual, el testimonio y la memoria se organizaron como prácticas específicamente resistentes (lo que no es así en cualquier otro caso). Es decir, se trata de una memoria signada políticamente desde sus inicios, aun cuando sus prácticas excedan lo exclusivamente político.

\footnotetext{
${ }^{1}$ Me refiero a la restricción de hecho que implica la polarización creciente en el acceso a los recursos económicos y sociales.
} 


\section{El testimonio}

\section{Conocimiento y experiencia}

El testimonio del sobreviviente relata una experiencia, $s u$ experiencia del terrorismo de Estado, de la que deviene un "saber" de eso que sólo a posteriori designamos como "terrorismo de Estado", pero que él reconoce como terror específico, inscrito sobre su cuerpo de manera cuidadosa y sistemática, mediante la tortura. El conocimiento de ese poder lo atraviesa aunque no encuentre "categorías" para nombrarlo teóricamente, tal vez porque la experiencia excede siempre a su propia conceptualización. Pero aún así sabe, sabe de otra manera y sabe otras cosas que quienes encuentran el nombre que finalmente designa el fenómeno, sin haberlo experimentado. ¿De qué clase de saber se trata?

Abordar esta cuestión nos remite a una discusión eminentemente epistemológica, que consiste en analizar la relación que existe entre experiencia y conocimiento.

Ya la fenomenología había enunciado, con Edmund Husserl, que en el mundo de vida, el yo reflexiona sobre sí por su experiencia, que le permite preguntarse qué y cómo, problematizándola. En consecuencia, la reflexión y la problematización se muestran como posibles y necesarias desde la experiencia del mundo cotidiano y no como funciones exclusivas del pensamiento teórico. Es más, Husserl señala al conocimiento experiencial como precientífico no sólo porque es previo a la ciencia sino también porque está presupuesto en ella, aunque la ciencia se arrogue una posición de superioridad y pretendida autonomía. En efecto, las ciencias construyen sobre el mundo de vida - una construcción de segundo grado, construcción de otra construcción diría más tarde Schutz, dentro de la misma vertiente fenomenológica- Esto no implica su supremacía en el proceso de conocimiento sino su reconocimiento 
como realidad primera, junto a la necesaria problematización de ambos, mundo de vida y ciencia, para hacerlos "trascendentalmente inteligibles".

Por su parte, Heiddegger afirmaba que el conocimiento parte de la articulación de una comprensión originaria, en el sentido de que

la comprensión que realmente abre el mundo es nuestra relación concreta con la cosa... la autenticidad es apropiación fundamentalmente en este sentido: se apropia de la cosa al relacionarse directamente con ella. Todo esto, aunque todavía vago, está sin embargo atestiguado claramente por nuestra experiencia común: hablar con conocimiento de causa sólo se puede cuando se ha experimentado de algún modo directo aquello de lo que se habla; y este experimentar nunca se entiende principalmente como un encuentro de un sujeto con un 'objeto', sino que se lo entiende como una relación más compleja, como la que se expresa, por ejemplo, en la expresión 'hacer una experiencia' o 'tener una experiencia'... El encuentro 'directo' con la cosa está pues vinculado con la apropiación de la cosa como tal. ${ }^{2}$

Desde esta perspectiva, la experiencia permite una apropiación que, sin ser suficiente, está en la base del conocimiento.

Con posterioridad, Alfred Schutz actualizó la visión fenomenológica para las ciencias sociales y, dando un paso más, se refirió a la relación entre la experiencia en el mundo de vida, su interpretación y la construcción de conocimientos socialmente compartidos. Señaló que la experiencia (que me sucede), el comportamiento (que tiene una intención) y la acción (que implica un proyecto) se comprenden desde esquemas interpretativos que, siendo individuales, han sido construidos intersubjetivamente, esto es, de manera social. Por su parte esos esquemas interpretativos constituyen acervos de conocimiento que operan en el mundo de vida y que parten de las

2 Vattimo 1996, 44. 
experiencias previas, ya sean propias o de otros, que nos las comunican. Así, las creencias, las expectativas y el conocimiento se forman como conocimientos socialmente performados que nuestra propia experiencia refina y modifica. Con ella apuntaba a la importancia de la experiencia y a la relevancia de lo socialcultural en la interpretación y asignación de sentido de ésta.

Más recientemente, Michel Maffesoli, en su reivindicación del conocimiento ordinario, propone pensar en la "insuperable relación que existe entre la experiencia y la ciencia de las cosas, entre lo vivido social y las representaciones que lo explican", ${ }^{3}$ vinculando de manera contundente conocimiento y experiencia. En esta misma dirección, propone "centrarse en el orden de la experiencia [que] remite una observación in statu nascendi con todas las dificultades conocidas y con la incertidumbre y la falta de conclusión inherentes a este procedimiento... 'mostración' como complemento de la clásica demostración".

Pero es Edgar Morin quien, desde la perspectiva del pensamiento complejo, no sólo desarrolla con mayor claridad la inseparabilidad de la experiencia del proceso de la reflexión sistemática que se desarrolla en el ámbito del conocimiento científico, sino que la propone como una de las garantías contra la pretensión de una racionalidad absoluta. En este sentido, piensa al método como un "movimiento impetuoso que va de la experiencia fenomenal a los paradigmas que organizan la experiencia". ${ }^{4} \mathrm{Y}$ advierte:

Tenemos necesidad de una racionalidad autocrítica, que pueda ejercer un comercio incesante con el mundo empírico, el único corrector del delirio lógico. El hombre tiene dos tipos de delirio. Uno es, evidentemente, bien visible, es el de la incoherencia absoluta, las onomatopeyas, las palabras pronunciadas al

${ }^{3}$ Maffesoli 1993, 93.

4 Apud Morin en Maffesoli 1993, 96. 
azar. El otro es mucho menos visible, es el delirio de la coherencia absoluta. El recurso contra este segundo delirio es la racionalidad autocrítica y la utilización de la experiencia. ${ }^{5}$

Edgar Morin abre así un doble resguardo: racionalidad autocrítica y utilización de la experiencia. Ambas se conectan en el lugar del sujeto. Allí confluyen lo empírico y lo trascendental, las experiencias del cuerpo y las de la cultura, el mundo de vida y el de la ciencia, realidades que la modernidad escindió y cuya crítica había realizado la fenomenología desde principios del siglo xx. Así pues, Morin señala la necesaria articulación entre experiencia y razón crítica, que obliga al doble rebasamiento de una en relación con la otra, resultando insuficientes per se pero también imprescindibles entre sí.

En este sentido el testimonio es capaz de conectar experiencia y razón crítica, es "conocimiento hecho experiencia de los que sí pueden recordar". 6

\section{Experiencia y verdad}

Sin duda el testimonio, como todo discurso, implica una "construcción" de la experiencia y no su "calca". Si tanto el testimonio como la teoría realizan construcciones, ¿en qué sentido puede haber en ellos una pretensión de verdad?

El discurso académico suele ser engañoso porque, en la mayor parte de los casos, desdibuja al sujeto que enuncia, creando la ilusión de que estamos frente a la sola "realidad" del sujetoobjeto enunciado. Por su parte, el testimonio podría ser igualmente engañoso, aunque en un sentido inverso. La primera persona puede sugerir la perfecta coincidencia entre el sujeto

${ }^{5}$ Morin 2000, 104.

${ }^{6}$ Vilanova 2006, 110. 
que enuncia y lo enunciado, una suerte de "calca" de uno sobre el otro que nos permitiría escapar de las posibles discrepancias y establecer una "verdad" última desde el "yo estuve ahî". Ambas son percepciones engañosas. Tanto el discurso científico como el testimonial son construcciones — de distinto orden- y en ambos se "sustrae", en el primer caso al sujeto que enuncia, en aras de una pretendida objetividad, en el segundo, por lo menos parte del sujeto que enuncia porque aun en el testimonio más completo, jamás está ni podría estar la totalidad del sujeto que enuncia ni, por supuesto, la totalidad del sujeto enunciado. Es decir, siempre hay alguna sustracción de la que dar cuenta.

Sin embargo, si se comparan uno y otro discurso, parece haber mayor pretensión de "verdad" precisamente en el discurso académico que no sólo describe sino que explica y analiza desde niveles importantes de generalización y objetivación.

El testimonio realiza un relato preciso, el de la propia experiencia, y al hacerlo fija de manera explícita sus límites. Parte invariablemente de la identificación del sujeto que enuncia, así como de la precisión de las coordenadas de tiempo y lugar en las que ocurrió la experiencia, lo que permite acotarlo de inmediato. Su obsesión por los detalles se explica en su propia incertidumbre, que lo lleva a hacer referencia constante a qué le pasó —y qué no-, qué vio u oyó - y qué no-- es decir, qué sabe y qué no sabe. Dado que tiene un relato que reconoce como incompleto, busca obsesivamente en él todo lo que pueda "dar de sí", todo lo que pueda ser significativo, más allá aun de la propia valoración. Es como si el testimonio pusiera y expusiera "todo", pero es un todo que se reconoce desde el inicio mismo de su presentación como fragmentario. En el caso específico de Argentina, los testimonios de los sobrevivientes no dicen, por ejemplo, "Los campos, concebidos como depósitos de cuerpos dóciles que esperaban 
la muerte, fueron posibles por la diseminación del terror". ${ }^{7}$ Ese nivel de generalización es propio del ensayo, de las ciencias sociales. Los testimonios dicen, en cambio: "Para nosotros fue la oscuridad total... No encuentro en mi memoria ninguna imagen de luz... Todo era noche y silencio. Silencio sólo interrumpido por los gritos de los prisioneros torturados y los llantos de dolor". ${ }^{8} \mathrm{El}$ "nosotros" se refiere a los prisioneros de La Perla, ${ }^{9}$ en esa situación específica, y describe cómo los cuerpos, en la oscuridad y entre los gritos, aterrados, esperaban la muerte.

Por lo regular el testimonio establece estas delimitaciones temporales y espaciales de la experiencia personal, vivida, que se piensa y articula desde coordenadas de sentido sociales, políticas, éticas. Recordar y entender son funciones inseparables, aunque hay distintos tipos de "entendimiento". El cuerpo que siente y la mente que piensa son inseparables entre sí e igualmente constitutivos de lo humano. No hay experiencia que no sea construida, es decir, pensada desde coordenadas de sentido sociales. En el mismo testimonio al que he hecho referencia, más adelante, la misma Graciela Geuna dice:

La realidad de La Perla era una realidad absoluta, total, con sus propias reglas. $\mathrm{Y}$ esa realidad comienza a imponerse con la venda y el proceso de aislamiento que desata: uno va encerrándose en sí mismo, se retrae y penetra cada vez más adentro de su conciencia. En esa situación uno se encuentra todo roto... La venda te lleva a tu interior y tu interior está destrozado y cada vez se fragmenta más hasta entrar en un mundo de categorías demenciales, irreales, donde todo lo que puede ser la vida está falseado y la propia vida es otra cosa. ${ }^{10}$

${ }^{7}$ Calveiro 2002, 94.

${ }^{8}$ Geuna, 20.

${ }^{9}$ La Perla es el nombre que se le dio a uno de los mayores campos de concentración de Argentina, que funcionó en la provincia de Córdoba.

${ }^{10}$ Geuna, 19. 
Geuna habla de su experiencia, pero lo que dice la trasciende. Las palabras que usa — "realidad total", "aislamiento", "estar todo roto", "fragmentarse"-, describen lo vivido con una fuerza reflexiva en la que la vivencia echa luz sobre la comprensión y viceversa.

A diferencia del discurso académico, el testimonio es, se sabe y se exhibe como fragmentario. Por eso reclama la multiplicidad, por eso hablamos de los testimonios en plural. Y la multiplicidad no remite a una especie de "sumatoria" para establecer verdades de carácter general. Por el contrario, la suma de testimonios permite identificar algunos ejes que los conectan entre sí evidenciando su veracidad, su confiabilidad, pero también nos enfrentan con una gran diversidad de situaciones distintas e incluso contradictorias, por ejemplo con respecto al tipo de vida dentro de unos campos y otros, a las características del personal militar, a las modalidades de la tortura, que remiten a la multidimensionalidad de un universo tan complejo como el concentracionario. En esa multiplicidad de los relatos no importan solamente los elementos comunes o compartidos que permitirían dar claves explicativas de carácter general. Más bien, lo contradictorio da cuenta de una realidad difícil de atrapar, que obliga a razonamientos no lineales. Por su parte, muchas veces es el dato único, la imagen especial que se vuelca en un único testimonio lo que "ilumina" parte del conjunto, lo que sin "aparecer" explícitamente en las otras experiencias, está sin embargo detrás de ellas como posible clave de sentido.

El testimonio expone frente a otros una verdad, "su" verdad, que reclama una cierta escucha y validación social para ser parte de "la" verdad socialmente constituida. Por ejemplo, algunos sobrevivientes relatan que sólo cuando su testimonio fue considerado como prueba para la condena de los comandantes, en el Juicio a las Juntas, sintieron que lo que les había pasado y lo que ya habían contado muchas veces, era realmente "verdadero". 
La multiplicidad del material testimonial permite seleccionar de él relatos muy diversos, capaces de "apoyar" construcciones distintas de lo concentracionario o, dicho en otros términos, cada testimonio conlleva una cierta construcción de la experiencia concentracionaria, no necesariamente coincidente con el resto. En este ámbito del sentido, de lo interpretativo, no es posible pensar que unas construcciones sean verdaderas y otras falsas sino que ofrecen ángulos diferentes donde, ciertamente, unos pueden ser más o menos interesantes, críticos, sugerentes que otros. Por ejemplo, el testimonio de Graciela Geuna es de una riqueza verdaderamente excepcional.

$\mathrm{Al}$ mismo tiempo, es importante distinguir entre distintos tipos de testimonio y creo que el aspecto principal para hacerlo es precisar frente a quién y en qué circunstancias fue realizado. Es sabido que todo discurso se estructura en relación con su destinatario. Esto marca una primera y obvia distinción entre los primeros testimonios hechos ante organismos de derechos humanos para la denuncia inicial, los que se hicieron con una voluntad incriminatoria en el marco de un proceso judicial, con la presencia física de los militares responsables, y los que se realizaron posteriormente mediante distinto tipo de entrevistas cuidadosas y mucho más detalladas. Porque, como lo señala Elizabeth Jelin, "ningún texto puede ser interpretado fuera de su contexto de producción y de su recepción, incluyendo las dimensiones políticas del fenómeno". ${ }^{11}$

Los conocedores de la entrevista, como Mercedes Vilanova, saben que la entrevista es una construcción hecha de a dos, en la que lo importante para el entrevistador es "escuchar la tonalidad, los aciertos y los dislates de la voz ajena, sobre todo cuando las personas se han mirado hacia adentro". ${ }^{12}$ Pero no

11 Jelin 2002, 91.

12 Vilanova 2006, 102. 
se trata sólo de la escucha sino que consiste más bien en un "diálogo", en una "intimidad creada a dos", que puede incluso ayudar al entrevistado a "descubrir" el pasado, lo que depende "de nuestra capacidad para generar confianza y empatía, para comprender y para participar con pasión en las experiencias que se nos relatan". ${ }^{13}$ En esa intimidad compartida, el entrevistador aporta, ayuda a rescatar del olvido, a encontrar nuevos hilos conductores de la historia y a hacer nuevas síntesis. Las preguntas, en el contexto de la conversación, pueden abrir las conexiones entre pasado, presente y futuro, así como las que existen entre la experiencia personal, la experiencia de un grupo de secuestrados o de militantes y la de toda una sociedad que, de distintas maneras, es contemporánea de lo narrado. En consecuencia, también es demasiado vago hablar sólo del testimonio, como si éste se redujera al que se produjo en oportunidad del Juicio a las Juntas. Hay distintas construcciones testimoniales de las que la existencia o no de la escucha, en primer lugar, y la calidad de ésta — no en un sentido técnico sino entendida más bien como calidad social de la escucha- son elementos sustanciales para la construcción de testimonios pertinentes, por llamarlos de alguna manera, y para que ellos sean parte de la construcción de una "verdad" socialmente reconocida como tal.

En este sentido, creo pertinente traer una reflexión de Paul Ricoeur en uno de sus últimos textos, aparecido en 1999 en la revista Historia y Grafía, donde se pregunta "en qué se distingue una reconstrucción de una construcción fantástica, incluso fantasiosa, es decir, en última instancia, de una ficción", ${ }^{14}$ y cómo se preserva la posición de lo real y pasado en la reconstrucción. Según Ricoeur, la historia se remite a la serie archivo-documento-huella, con lo que permanece atrapada en el antiguo enigma del desciframiento último de la huella. Frente a

13 Vilanova 2006, 103.

${ }^{14}$ Ricoeur 1999, 164. 
ello, Ricoeur propone que el testimonio, estructura de transición entre memoria e historia, es capaz de resolver el enigma de la huella y su desciframiento.

Sustituyendo a la impronta, el testimonio desplaza la problemática de la huella; es necesario pensar la huella a partir del testimonio y no a la inversa... Es necesario dejar de preguntarse si una narración se asemeja a un acontecimiento; más bien hay que preguntarse si el conjunto de los testimonios, confrontados entre sí, es fiable. Si es éste el caso, podemos decir que el testigo nos hizo asistir al acontecimiento relatado. ${ }^{15}$

Como es evidente, en el caso argentino, el derecho procedió de esta manera. En el Juicio a las Juntas, se tomó el conjunto de los testimonios, se los confrontó y, dada su consistencia, se los consideró fiables para acreditar la "verdad" jurídica de ciertas acusaciones y no de otras. Se impone entonces una pregunta: ¿por qué la historia no habría de proceder de manera semejante? ¿Por qué los testimonios representan una verdad para el derecho, la política y la reparación moral, que estaría en duda a la hora de construir el relato histórico? ¿Qué extraterritorialidad se supone en la ciencia?

\section{Testimonio, memoria e historia}

Decíamos que el testimonio es una construcción reflexiva de una experiencia particular, la del sobreviviente, con capacidades distintas de descripción e interrogación, según sea el caso. No da cuenta más que de esa experiencia y no tendría por qué hacerlo; ése es su sentido. El testigo atestigua desde su presencia en el lugar de los acontecimientos. El conjunto de los

15 Ricoeur 1999, 165. 
testimonios, no obstante, permite establecer "verdades" jurídicas y, de alguna manera, "asistir" a los acontecimientos relatados, desde el lugar de la víctima.

La memoria del terrorismo de Estado ha comprendido una serie de prácticas, una de las cuales fue la recopilación de los testimonios. De hecho, "la misma constitución del archivo (de testimonios de sobrevivientes) es un acto de memoria y una contribución a la memoria colectiva sobre los alcances del terror estatal". ${ }^{16}$ Por ello, se podría decir, de manera más general, que el trabajo de la memoria ha consistido en una práctica de resistencia frente al poder desaparecedor del Estado. Se ha abocado a hacer evidentes las marcas del terrorismo estatal sobre la sociedad, recuperando las voces de los agraviados y forzando la "reaparición" de lo que se pretendía desaparecido. En este sentido, debe dar lugar a una multiplicidad de miradas distintas e incluso contradictorias pero todas resistentes. No hay dueños de la memoria pero esta apertura es posible dentro de un posicionamiento específico en las relaciones de poder pasadas y presentes: es parte de las resistencias y ése es su sentido.

"El discurso de la memoria y el de la historia son hermanos". ${ }^{17}$ Es cierto que la historia busca un relato explicativo que no carece de posicionamiento. Sin embargo, debe dar cuenta de los diferentes sentidos que se manifiestan en una sociedad, no sólo de los resistentes. Se podría decir que se trata de un relato más abarcador, de mayor generalidad, que debe dar cuenta de procesos en los que el testimonio o la memoria no tienen por qué internarse. Pero además de estas distinciones formales en el tipo de construcción, el relato histórico "fija" la versión oficial, "cuajando" la memoria social y, en este sentido, tiene una dimensión extraordinariamente política. Es por ello que, a

${ }^{16}$ Carnovale et al. 2006, 43.

17 Vilanova 2006, 92. 
la hora de su construcción, la relación que guarde con la memoria y con el testimonio será decisiva; de ello dependerá el papel que tendrán los agraviados, los otros, en su relato.

Pero en cualquiera de estos discursos hay dos cuestiones fundamentales que, a mi entender, se vinculan con una cierta postura ética: la responsabilidad y la capacidad de apertura al Otro que son los otros. Ambas son necesarias en el acto de testimoniar y de hacer memoria o historia.

Se podría decir que una postura ética en el testimonio implica reflexionar sobre las propias responsabilidades en lugar de construir discursos autojustificatorios, así como tener conciencia del otro que escucha y darle un lugar de consideración, es decir, hacer un relato abierto, que le permita "ser parte", que no se encripte en el propio sufrimiento sino que trace "puentes" para facilitar la comprensión y la transmisión. La transmisión no sólo es posible sino que está ocurriendo de manera constante, independientemente de nuestra voluntad, pero poner la experiencia sobre la mesa, exponerla ante el otro - que es quien decide lo que toma o desecha de ella-, es un acto de apertura porque le abre un lugar en esa historia. A su vez, el interés por la transmisión conlleva el cuestionamiento de las propias explicaciones, su puesta entre paréntesis, y la consideración de otras formas de entender lo vivido, que permiten al sobreviviente ser parte activa del procesamiento social y no su lastre.

Los trabajos de memoria exceden en mucho lo testimonial. Sin duda comprenden la recuperación y la organización de dicho material, pero también la preservación de espacios físicos y simbólicos de la memoria, las distintas acciones para la identificación, el juicio y el castigo de los responsables, las prácticas educativas para el "pasaje" social de lo vivido, en fin, un haz de acciones colectivas que recogen, interpretan y procesan lo experimentado colectivamente desmontando tanto el silencio como los discursos del Estado para dar paso a la visión de las víctimas, de los vencidos, de los otros. Por la diversidad 
de perspectivas que comprende es inapropiable por uno $\mathrm{u}$ otro sector. Necesariamente excede a cualquiera de los numerosos protagonistas que cuentan, piensan, elaboran y transmiten, donde sea que estén parados para, en cambio, dar espacio a los distintos "armados" de la memoria, a su diversidad.

Por último, una construcción histórica que dé cuenta de las deudas con el pasado, reclama la apertura del discurso académico, e incluso su puesta entre paréntesis momentánea, para darle un lugar a las voces de los actores, a sus prácticas y a los sentidos que enuncian. Sin renunciar a la construcción propia de las ciencias sociales, que es parte de su responsabilidad, parece necesario abandonar la postura de calificación y jerarquización de los saberes, como otra parte de su propia responsabilidad. En este contexto, la apertura implica articularse con esos otros saberes en lugar de oponerlos, reconociendo el enorme valor de los trabajos de la memoria para la construcción de un relato histórico en el que la densidad de lo vivido en el pasado permita cierta "iluminación" del futuro.

\section{REFERENCIAS}

Calveiro, Pilar, 2002. Desapariciones, México, Taurus.

Carnovale, Vera, 2006. Federico Lorenz y Roberto Pittaluga (comps.), Historia, memoria y fuentes orales, Buenos Aires, Cedinci.

Geuna, Graciela, s./f. Testimonio, Buenos Aires, Comisión Argentina de Derechos Humanos, mimeo.

Jelin, Elizabeth, 2002. Los trabajos de la memoria, Madrid, Siglo XXI.

MAFFesoli, Michel, 1993. El conocimiento ordinario, México, FCE. MoRIN, Edgar, 2000. Introducción al pensamiento complejo, Barcelona, Gedisa.

Ricoeur, Paul, 1999. "La marca del pasado", Historia y Grafía, 13, México, Universidad Iberoamericana. 
Schutz, Alfred, 1995. El problema de la realidad social, Buenos Aires, Amorrortu.

VAтTIMO, Gianni, 1996. Introducción a Heidegger, Barcelona, Gedisa. Vilanova, Mercedes, 2006. "Rememoración y fuentes orales", en Vera Carnovale, Federico Lorenz y Roberto Pittaluga (comps.), Historia, memoria y fuentes orales, Buenos Aires, Cedinci. 